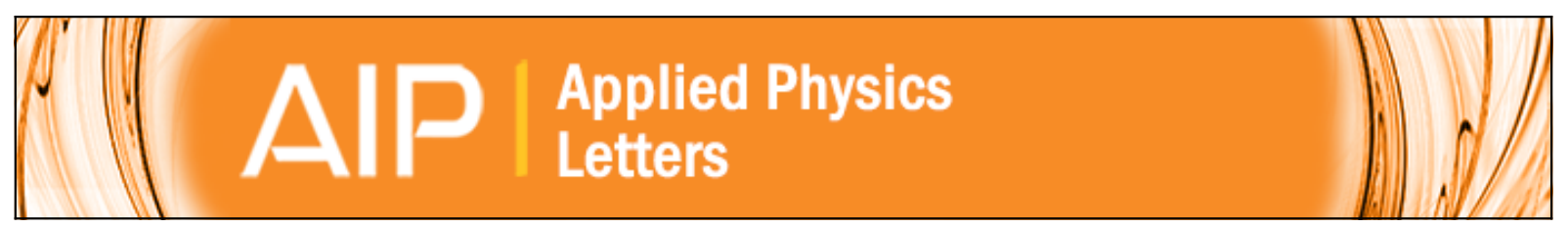

\title{
Faceting oscillations in nano-ferroelectrics
}

J. F. Scott and Ashok Kumar

Citation: Applied Physics Letters 105, 052902 (2014); doi: 10.1063/1.4892362

View online: http://dx.doi.org/10.1063/1.4892362

View Table of Contents: http://scitation.aip.org/content/aip/journal/apl/105/5?ver=pdfcov

Published by the AIP Publishing

\section{Articles you may be interested in}

Determining ferroelectric polarity at the nanoscale

Appl. Phys. Lett. 98, 052904 (2011); 10.1063/1.3549300

Scaling of structure and electrical properties in ultrathin epitaxial ferroelectric heterostructures

J. Appl. Phys. 100, 051609 (2006); 10.1063/1.2337363

Density inhomogeneity in ferroelectric thin films

Appl. Phys. Lett. 89, 052901 (2006); 10.1063/1.2244045

Misfit dislocations in nanoscale ferroelectric heterostructures

Appl. Phys. Lett. 86, 192910 (2005); 10.1063/1.1922579

Ferroelectric epitaxial nanocrystals obtained by a self-patterning method

Appl. Phys. Lett. 83, 2211 (2003); 10.1063/1.1611258

Want to publish your paper in the \#1 MOST CITED journal in applied physics?

With Applied Physics Letters, you can.

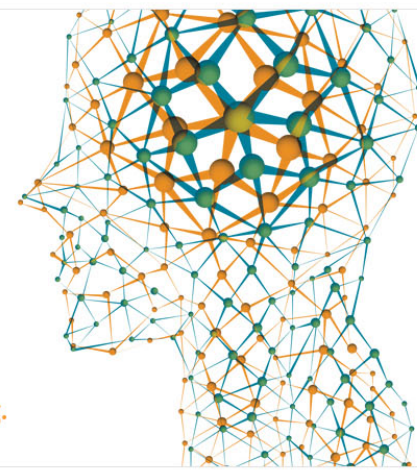




\title{
Faceting oscillations in nano-ferroelectrics
}

\author{
J. F. Scott ${ }^{1, a)}$ and Ashok Kumar ${ }^{2, a)}$ \\ ${ }^{1}$ Cavendish Laboratory, Cambridge University, Cambridge, United Kingdom \\ ${ }^{2}$ CSIR-National Physical Laboratory, Delhi, India
}

(Received 2 July 2014; accepted 23 July 2014; published online 4 August 2014)

\begin{abstract}
We observe periodic faceting of 8-nm diameter ferroelectric disks on a $10 \mathrm{~s}$ time-scale when thin $\mathrm{Pb}\left(\mathrm{Zr}_{0.52} \mathrm{Ti}_{0.48}\right) \mathrm{O}_{3}$ film is exposed to constant high-resolution transmission electron microscopy beams. The oscillation is between circular disk geometry and sharply faceted hexagons. The behavior is analogous to that of spin structure and magnetic domain wall velocity oscillations in permalloy [Bisig et al., Nat. Commun. 4, 2328 (2013)], involving overshoot and de-pinning from defects [Amann et al., J. Rheol. 57, 149-175 (2013)]. (C) 2014 AIP Publishing LLC.
\end{abstract}

[http://dx.doi.org/10.1063/1.4892362]

Historically, ferroelectric domains have been treated in analogy with magnetic domains. Although the two have some superficial similarities with regard to static structure, their dynamics is fundamentally different in two important ways: First, the temporal dependence of spin waves is described by the Landau-Lifshitz-Gilbert Equations, and these equations are first-order in time. That requires that when the external magnetic field (H) stops, the spin precession stops instantly. By comparison, ferroelectric polarizations and domain walls obey Newton's Laws, and in particular are second-order in time; this implies momentum, and ferroelectric walls coast long distances (ca. microns) after the external electric field (E) are terminated. Second, because magnetic domain walls carry no mass, they can readily be accelerated to supersonic speeds, as shown by Demokritov et al., ${ }^{1,2}$ at which point they emit coherent acoustic phonons at angles analogous to Cerenkov radiation or bow waves; by comparison, ferroelectric domain walls carry mass and cannot be supersonic without causing shock waves and fracture.

It has been known that ferroelectrics under highresolution transmission electron microscopy (HRTEM) studies respond to the e-beam irradiation by significant restructuring of their domains and domain walls, ${ }^{3,4}$ but it has not been completely clear whether this is driven thermally by beam heating and the thermal conductivity anisotropy of the target or by charging and depolarization fields. In this context, it is very important to compare faceting under HRTEM with faceting observed in atomic piezo-force microscopy (PFM), since the latter does not involve the same degree of sample heating. Ganpule et al. reported a situation in lead zirconate titanate nano-structures, which is probably due to thermal anisotropy along [111] axes. ${ }^{5}$ Similar hexagonal faceting was first seen in the famous Schwartz-Hora Effect ${ }^{6}$ and in related experiments in which laser beams produce hexagonal distributions of charged defects that fill space. ${ }^{7,8}$ Hexagonal faceting also occurs with foams and surfactants (viscous fingering), due probably to thermal anisotropy of the substrates; but only twofold symmetry instabilities occur in magnetic bubble domains (circular to elliptical). A short

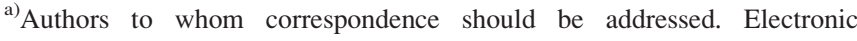
addresses: jfs32@cam.ac.uk and ashok553@nplindia.org
}

pedagogic review has been given by one of the present authors, ${ }^{9}$ and a more detailed analysis of hexagonal faceting in polyvinylidene fluoride (PVDF) films is by Lukyanchuk et al. ${ }^{10}$

The present work seems especially interesting because the nano-crystals examined have thicknesses of ca. $80 \mathrm{~nm}$ which are $\ll$ the length $(1-3 \mu \mathrm{m})$ of the multi-walled nanotubes on which they are mounted, and in this respect approximate low-dimensional systems. As Berge et al. have emphasized, ${ }^{11}$ although three-dimensional crystals are usually faceted, faceting is not permitted at thermal equilibrium in two dimensions ${ }^{12}$ because the perimeter of a two dimensional [2D] structure is one-dimensional and cannot exhibit long-range order at finite temperatures. ${ }^{13}$ But $[2 \mathrm{D}]$ faceting can occur dynamically during growth processes and has been modeled numerically. ${ }^{14,15}$ It is worth noting that unfaceted domains have been known in ferroelectrics for more than fifty years, with Cameron reporting circular "lake-like" domains in tetragonal $\mathrm{BaTiO}_{3}$ in 1957. ${ }^{16}$

It is also important to comment on why only hexagonal faceting is observed, and not pentagons or heptagons, etc. Since the samples are single isolated films, macroscopic space-filling is not a criterion, but domain wall orientation inside the film is a criterion. Hence, there may be a relationship to the formation of foams from bubbles. Let is consider each nucleating nano-domain as analogous to a bubble, pressed nearly flat against a substrate. Only three walls meet along a line, at angles of $120^{\circ}$ due to surface tension equality. Only four walls can meet at a point, at angles of $\cos ^{-1}(-1 / 3) \approx 109.47^{\circ}$. All these rules, known as Plateau's laws, determine how a foam is built from bubbles. Indeed, the formation of hexagonal facets in foams is well known. ${ }^{17}$

Our studies were carried out with a high-resolution Cs-probe corrected HRTEM (Model: JEOL JEM-2200FS) system, operated at a $200 \mathrm{kV}$ voltage $(\sim 200 \mathrm{keV}$ kinetic energy) with $0.5 \mathrm{~A} / \mathrm{m}$ probe current density in order to minimize the damage rate due to Bethe-Bloch cross-section for electron-electron interaction. During the investigation, the $\mathrm{Pb}\left(\mathrm{Zr}_{0.52} \mathrm{Ti}_{0.48}\right) \mathrm{O}_{3}$ (PZT) nanoparticles were first exposed to electron beams for an hour to get stabilized; later the images were recorded in continuous mode with the interval of $10 \mathrm{~s}$. The PZT films were $50-80 \mathrm{~nm}$ thick, deposited on multiwalled carbon nanotubes which in turn were on $\mathrm{n}-\mathrm{Si}$ 
substrates. The length of each nanotube was ca. 1-3 $\mu \mathrm{m}$ depending on growth conditions. ${ }^{18}$ HRTEM studies were carried out on the PZT thin film coated multi-wall carbon nanotubes (MWCNT). Faceting behavior of PZT domains were investigated near the edge portion to maintain boundary conditions (ca. films thickness $\ll$ peripheral area).

The observed faceting is global, but it is more readily seen at the edge of nanocrystals grown in the island growth common in polycrystalline $\mathrm{PZT}$ or $\mathrm{BaTiO}_{3}$. The geometry is shown in Fig. 1. In most instances, the faceting was highly hexagonal with $120^{\circ}$ angles between two (111) planes; however, (110) faces were also faceted. On some occasions, pentagonal facets or square facets were observed. Note that the stripe domains are predominantly orthogonal to the edges in this figure. Fig. 1 illustrates the domain structure at $t=0$ (e-beams turned on). Notice in the boxed region of Fig. 1(a) that there is a generally round disk shape for the PZT nanocrystal, and that configurations the stripe domains inside the crystal are mostly normal to the outer hexagonal edge.

Fig. 2(a-1) illustrates the HRTEM images (both real and reciprocal space image side by side) of same target obtained in the interval of 10, 20, 30, 40, 50, and $60 \mathrm{~s}$. Image taken in the next shot at $\mathrm{t}=10 \mathrm{~s}$ (Figs. 2(a) and 2(b)) shows the internal stripe domains that realigned predominantly parallel to the outer edge, and the hexagonal faceting is more pronounced. This suggests that the internal domain realignment controls the external faceting; inspection of transmission electron microscope (TEM) micrographs reveals that closure vertex domain structures evolve into stripe domains parallel to the external facets. Figs. 2(c) and 2(d) show the TEM image at $\mathrm{t}=20 \mathrm{~s}$ and Figs. 2(e) and 2(f) show the TEM image at $\mathrm{t}=30 \mathrm{~s}$. In this condition, we see internal domain realignment and less distinct hexagonal faceting. Figs. 2(g) and 2(h) at $\mathrm{t}=40 \mathrm{~s}$ show stripe domains, in this situation (110) faces are almost orthogonal to each other. With further imaging at $50 \mathrm{~s}$ (Figs. 2(i) and 2(j)), we observed the faceting of (110) faces. Finally, by imaging of same crystal at $\mathrm{t}=60 \mathrm{~s}$, we observe reversion to hexagonal faceting and

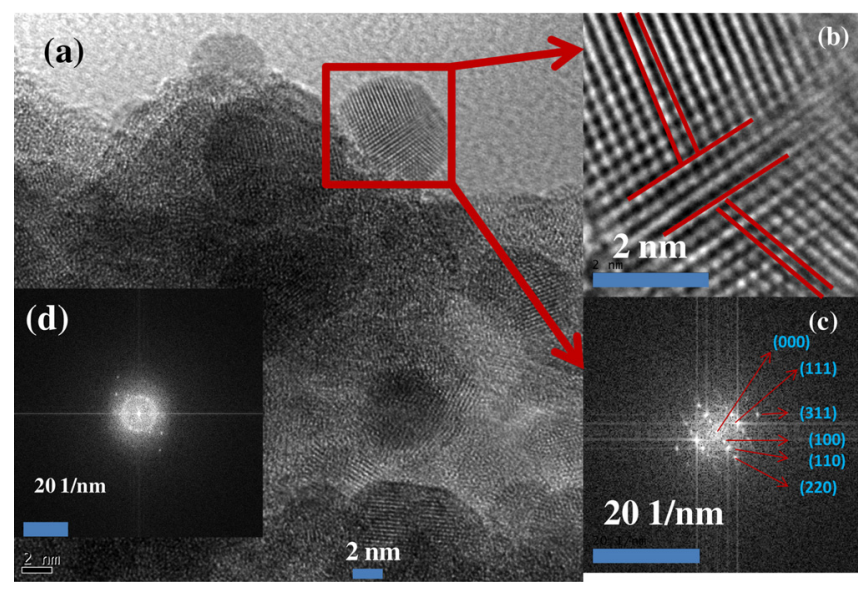

FIG. 1. Outer edge HRTEM images of thick PZT thin films (average $50-80 \mathrm{~nm}$ conformal coating of PZT on 1-3 $\mu$ m length MWCNT): (a) Large area HRTEM image, and HRTEM image of PZT nanocrystals of sizes $5-8 \mathrm{~nm}$ (red box), (b) faceting of lattice plane, Inverse Fast Fourier Transform (IFFT) images, (c) Fast Fourier Transform (FFT) image with assigned crystal plane, (d) FFT image of large area PZT thin films. internal stripe domains well aligned parallel to the outer edges of the sample.

To check the universal nature of faceting, similar experiment was carried out on another target; interestingly, it shows clean hexagonal faceting after continuous irradiation of e-beams. Fig. 3 shows progress of domain faceting with time. It starts with (111) parallel plane at $\mathrm{t}=0 \mathrm{~s}$, surprisingly we see $120^{\circ}$ reversal of plane in next imaging time $(t=10 \mathrm{~s})$.

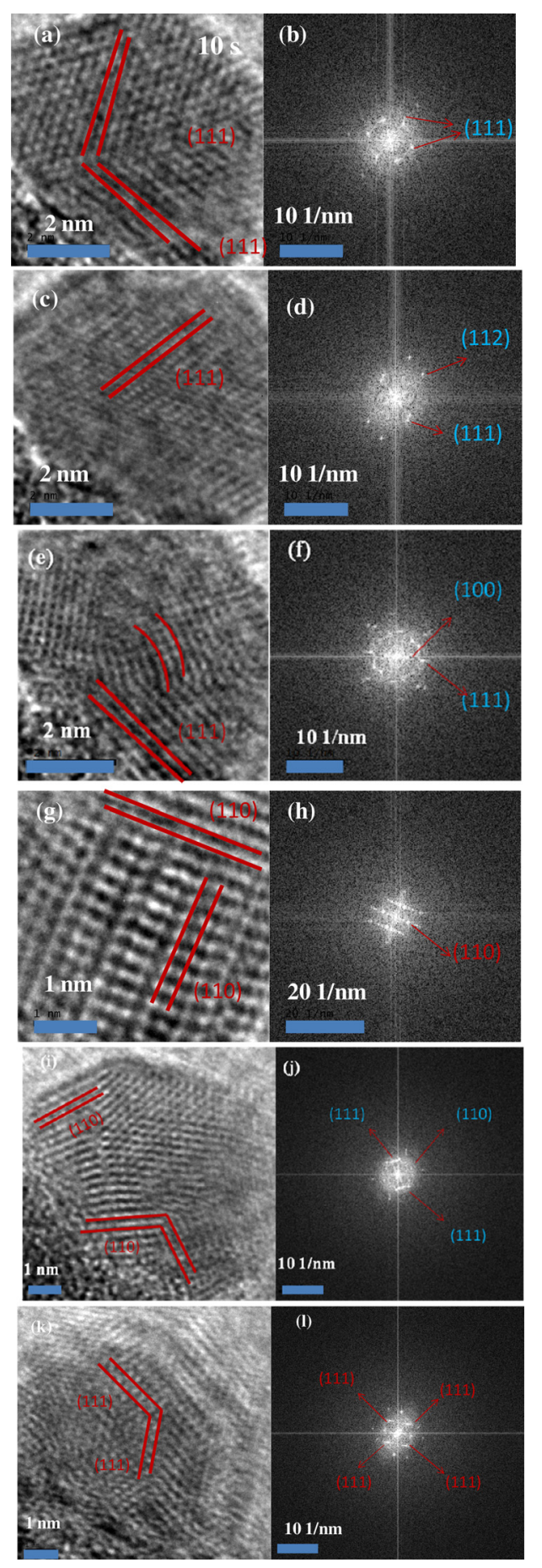

FIG. 2. (a-1) shows IFFT images and FFT images of same PZT nano-crystals (red box in Fig. 1) in different time scale (10-60 s). Assigned crystal planes and their orientations are given in each figure. TEM Images were taken under the continuous irradiation of e-beams at $10 \mathrm{~s}$ (Figs. (a) and (b)), $20 \mathrm{~s}$ (Fig. (c) and (d)), 30 s (Figs. (e) and (f)), 40 s (Figs. (g) and (h)), $50 \mathrm{~s}$ (Figs. (i) and (j)), and $60 \mathrm{~s} \mathrm{(Fig.} \mathrm{(k)-1)),} \mathrm{with} \mathrm{increasing} \mathrm{time} \mathrm{scale} \mathrm{from} \mathrm{top} \mathrm{to}$ bottom. 

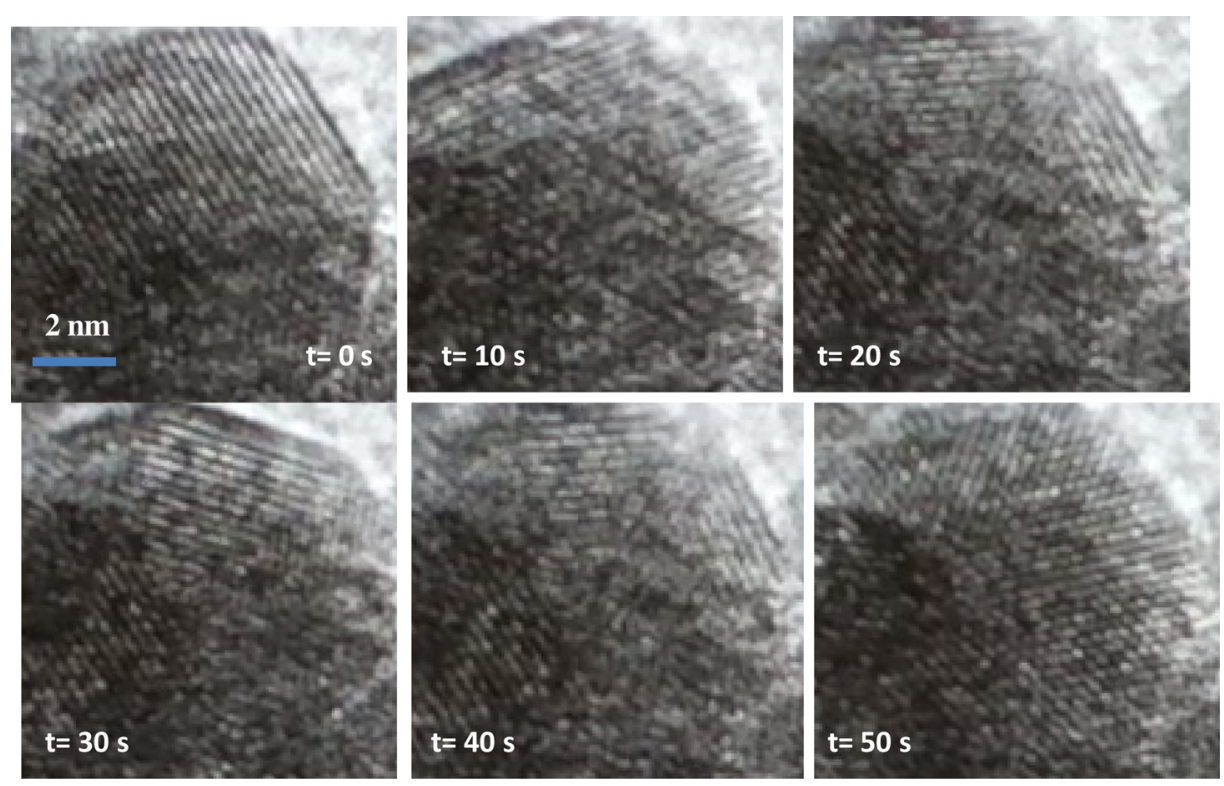

FIG. 3. Shows HRTEM images of other PZT target in different time scale $(0-50 \mathrm{~s})$. Average size of this crystal is around 7-9 nm. Evolution and restructuring of hexagonal faceting is clearly visible with time.
Image taken in $20 \mathrm{~s}$ is rather more clear with evolution of (110) planes orthogonal to the hexagonal facets of (111) planes. TEM image taken in $30 \mathrm{~s}$ shows the disappearance of (110) planes with clear picture of hexagonal faceting. Images obtained at $40 \mathrm{~s}$ and $50 \mathrm{~s}$ suggest further realignments of planes with interaction of energetic e-beams. The evolution of hexagonal faceting and its realignments are natural, and it evolves and disappears with time, however it is not obvious that it appears and destroys with a definite interval of time.

Until the past few months, domain wall oscillations in which wall velocities actually change sign were neither observed nor predicted. However, very recently Bisig et al. reported ${ }^{19}$ changes in the sign of magnetic domain wall velocity under applied magnetic fields on a very short timescale $(100 \mathrm{~ns})$ in permalloy disks of comparable geometry to the ferroelectrics in our study $(50-80 \mathrm{~nm}$ thick; $1.0 \mu \mathrm{m}$ radius). It is important in that work (especially their Fig. 5) that the wall velocities actually reverse the direction. This is interpreted as overshoot in the radial wall velocities as the domain configurations transform from vertex cores to transverse domain (stripe) walls. They record a $50 \mathrm{~ns}$ oscillation, about $200 \times 10^{6}$ times slower than in our work. These data seem analogous to ours despite the large difference in timescale, because we have independent evidence of both vertex (and vortex) structures in our samples ${ }^{20}$ and of radial electric fields ${ }^{3}$ caused by TEM charge injection. Of course the anticipated time scale for ferroelectric wall motion, involving creep velocities of typically $10^{-10} \mathrm{~m} / \mathrm{s}$ and real mass transport, will be much slower than for spin propagation. ${ }^{21}$

In general, strain overshoot in materials requires viscosity and is a topic of current interest. ${ }^{22}$ We note that in the paper by Amann et al. $^{22}$ the characteristic relaxation time for their viscoelastic materials was about $1 \mathrm{~min}$, as in the present work. We have no independent theoretical estimate of this time for our domain walls, but this comparison shows that it is similar to that in liquid crystals, which is plausible in view of the initial domain-wall topology comparison with nematics of Srolovitz and Scott. We know in the present work that the driving force for faceting is not thermal: Heating is only about $1 \mathrm{~K}^{23}$ The actual driving force is charging (Ahluwalia and $\mathrm{Ng}$ ), and its effect upon surface tension. The surface tension in ferroelectric nanodomains has been analyzed by Lukyanchuk et $a .^{24}$ and shown very recently by $\mathrm{Scott}^{25}$ to fit quantitatively hoop stress (neglected in all previous models, such as that of $\mathrm{Arlt}^{26}$ ). The fact that domain wall motion in ferroelectric films can be treated as ballistic motion in a viscous medium was demonstrated clearly by Dawber et $a l .{ }^{27} \mathrm{We}$ emphasize also that the preference for hexagon facets probably arises from the underlying lattice symmetry here with [111] axes playing a role. Although hexagonal symmetry of facets is also known for crystals with only twofold symmetry, ${ }^{10}$ strongly hexagonal faceting is observed in hexagonal magnesium $(\mathrm{Mg})$ nanopores under HRTEM irradiation ${ }^{23}$ and is registered along crystallographic axes; and Tegze et $a l .{ }^{28}$ recently report strongly hexagonal fingering in amorphous fluids (as is well known previously).

In the particular case of PZT thin-film disks, $\mathrm{Ng}$ et al. ${ }^{29}$ have given a detailed model of the role of fringing fields, emphasizing that they behave quite differently for atomic force microscopy (AFM) geometries (point-like top electrode) and parallel-plate geometries, and that the $180^{\circ}$ switching observed often proceeds via a two-step process involving $90^{\circ}$ domains. Although the HRTEM geometry resembles AFM in the sense that there is a radial field generated by a central charge injection, the TEM beam diameter is very different from that of an AFM tip, so that different dynamics should result in these two situations. Although more detailed calculations are required, it appears that fringing fields and boundary conditions play a key role; indeed, faceting oscillations are not observed in square or triangular PZT targets. ${ }^{30}$

Dr. Ashok Kumar (A.K.) would like to thank Professor R S Katiyar, Dr. Margarita Correa and Mr. Oscar Resto for acquiring in HRTEM data.

${ }^{1}$ S. O. Demokritov, A. I. Kirilyuk, N. M. Kreines, V. I. Kudinov, V. B. Smirnov, and M. V. Chetkin, JETP Lett. 48, 294 (1988). 
${ }^{2}$ S. O. Demokritov, A. I. Kirilyuk, N. M. Kreines, V. I. Kudinov, V. B. Smirnov, and M. V. Chetkin, J. Magn. Magn. Mater. 102, 339 (1991).

${ }^{3}$ R. Ahluwalia, N. Ng, A. Schilling, R. McQuaid, D. J. Srolovitz, D. M. Evans, J. M. Gregg, and J. F. Scott, Phys. Rev. Lett. 111, 165702 (2013).

${ }^{4}$ A. W. Robertson, C. S. Allen, Y. A. Wu, K. He, J. Oliver, J. Neethling, A. I. Kirkland, and J. H. Warner, Nat. Commun. 3, 1144 (2012).

${ }^{5}$ C. S. Ganpule, A. L. Roytburd, V. Nagarajan, J. F. Scott, and R. Ramesh, Phys. Rev. B 65, 014101 (2002).

${ }^{6}$ H. Schwarz and H. Hora, Appl. Phys. Lett. 15, 349 (1969).

${ }^{7}$ J. F. Scott and R. A. O'Sullivan, Nature 382, 305 (1996).

${ }^{8}$ J. F. Scott and R. A. O'Sullivan, Physica A 233, 655 (1996).

${ }^{9}$ J. F. Scott, EPL 103, 37001 (2013).

${ }^{10}$ I. Lukyanchuk, P. Sharma, T. Nakajima, S. Okamura, J. F. Scott, and A. Gruverman, e-print arXiv:Cond-mat/1309.0291.

${ }^{11}$ B. Berge, L. Faucheux, K. Schwab, and A. Libchaber, Nature 350, 322 (1991).

${ }^{12}$ G. Gallavotti, Commun. Math. Phys. 27, 103 (1972).

${ }^{13}$ L. Landau and E. Lifshitz, Statistical Physics (MIR, Moscow, 1951).

${ }^{14}$ R. Savit and R. Ziff, Phys. Rev. Lett. 55, 2515 (1985).

${ }^{15}$ P. Meakin, Phys. Rev. A 38, 418 (1988).

${ }^{16}$ D. P. Cameron, IBM J. Res. Dev. 1, 2 (1957).

${ }^{17}$ J. M. Flesselles, M. O. Magnasco, and A. Liebchaber, Phys. Rev. Lett. 67, 2489 (1991).
${ }^{18}$ A. Kumar, S. G. Shivareddy, M. Correa, O. Resto, Y. Choi, M. Cole, R. S. Katiyar, J. F. Scott, G. A. J. Amaratunga, H. Lu, and A. Gruverman, Nanotechnology 23, 165702 (2012).

${ }^{19}$ A. Bisig, M. Stärk, M. A. Mawass, C. Moutafis, J. Rhensius, J. Heidler, F. Büttner, M. Noske, M. Weigand, S. Eisebitt, T. Tyliszczak, B. V. Waeyenberge, H. Stoll, G. Schütz, and M. Kläui, Nat. Commun. 4, 2328 (2013).

${ }^{20}$ L. J. McGilly and J. M. Gregg, Nano Lett. 11, 4490 (2011).

${ }^{21}$ P. Paruch, T. Giamarchi, T. Tybell, and J.-M. Triscone, J. Appl. Phys. 100, 051608 (2006).

${ }^{22}$ C. P. Amann, M. Siebenbürger, M. Krüger, F. Weysser, M. Ballauff, and M. Fuchs, J. Rheol. 57, 149 (2013).

${ }^{23}$ H. Zheng, Y. Liu, F. Cao, S. Wu, S. Jia, A. Cao, D. Zhao, and J. Wang, Sci. Rep. 3, 1920 (2013).

${ }^{24}$ I. A. Lukyanchuk, A. Schilling, J. M. Gregg, G. Catalan, and J. F. Scott, Phys. Rev. B 79, 144111 (2009).

${ }^{25}$ J. F. Scott, J. Phys.: Condens. Matter 26, 212202 (2014).

${ }^{26}$ G. Arlt, J. Mater. Sci. 25, 2655 (1990).

${ }^{27}$ M. Dawber, D. J. Jung, and J. F. Scott, Appl. Phys. Lett. 82, 436 (2003).

${ }^{28}$ G. Tegze, G. I. Tóth, and L. Gránásy, Phys. Rev. Lett. 106, 195502 (2011).

${ }^{29}$ N. Ng, R. Ahluwalia, H. B. Su, and F. Boey, Acta Mater. 57, 2047 (2009).

${ }^{30}$ A. Gruverman, D. Wu, H. J. Fan, I. Vrejoiu, M. Alexe, R. J. Harrison, and J. F. Scott, J. Phys.: Condens. Matter 20, 342201 (2008). 